\title{
Candida Surveillance in Surgical Intensive Care Unit (SICU) in a Tertiary Institution
}

\author{
Yi Xin Liew ${ }^{1 \dagger}$, Jocelyn Teo ${ }^{1 \dagger}$, Irene Ai-Ling Too ${ }^{2}$, Cecilia Cheng-Lai Ngan ${ }^{3}$, Ai Ling Tan ${ }^{3}$, Maciej Piotr Chlebicki ${ }^{4}$,
} Andrea Lay-Hoon Kwa ${ }^{1,56^{*}}$ and Winnie Lee ${ }^{1}$

\begin{abstract}
Background: Colonization of patients occurs before development into invasive candidiasis. There is a need to determine the incidences of Candida colonization and infection in SICU patients, and evaluate the usefulness of beta-D-glucan (BDG) assay in diagnosing invasive candidiasis when patients are colonized.

Methods: Clinical data and fungal surveillance cultures in 28 patients were recorded from November 2010, and January to February 2011. Susceptibilities of Candida isolates to fluconazole, voriconazole, amphotericin B, micafungin, caspofungin and anidulafungin were tested via Etest. The utilities of BDG, Candida score and colonization index for candidiasis diagnosis were compared via ROC.

Results: 30 BDG assays were performed in 28 patients. Four assay cases had concurrent colonization and infection; 23 had concurrent colonization and no infection; three had no concurrent colonization and infection. Of 136 surveillance swabs, 52 (38.24 \%) were positive for Candida spp, with C. albicans being the commonest. Azole resistance was detected in C. albicans (7\%). C. glabrata and C. tropicalis were, respectively, 100 and 7 \% SDD to fluconazole. All 3 tests showed high sensitivity of $75-100 \%$ but poor specificity ranging $15.38-38.46 \%$. BDG performed the best (AUC of 0.89).
\end{abstract}

Conclusions: Despite that positive BDG is common in surgical patients with Candida spp colonization, BDG performed the best when compared to $\mathrm{Cl}$ and $\mathrm{CS}$.

Keywords: Beta-D-glucan, Candida surveillance, Colonization, Surgical intensive care unit

\section{Background}

Invasive candidiasis (IC) in critically ill patients is associated with high mortality and morbidity [1,2]. While early diagnosis and treatment of IC will improve survival rates significantly, clinical and radiological signs are non-specific and develop late in the course of the disease. Furthermore, the epidemiology of Candida infections have evolved, with an increased proportion of non-albicans due to frequent use of azoles [3]. This in turn affects the efficacy of the empiric antifungal regimen used in these high risk patients who are critically ill. Therefore, it is important to understand the epidemiology

\footnotetext{
* Correspondence: andrea.kwa.l.h@sgh.com.sg

${ }^{\dagger}$ Equal contributors

'Department of Pharmacy, Singapore General Hospital, Block 8 Level 2, Outram Road, Singapore 169608, Singapore

${ }^{5}$ Emerging Infectious Diseases, Duke-National University of Singapore Graduate Medical School, 8 College Rd, Singapore 169857, Singapore Full list of author information is available at the end of the article
}

and incidence of Candida colonization and infections in a surgical intensive care unit setting, where the patients are at higher risks for IC, in order for effective treatment to be instituted early [4].

Rendering prompt antifungal therapy is also limited by current culture-based diagnostic techniques for the diagnosis of Candida bloodstream infection which lack sensitivity, with up to $50 \%$ of cases remaining undiagnosed. Moreover, these tests have long lag-time, ranging from 85 to $124 \mathrm{~h}$ [5]. Hence, early detection of a component of fungal cell wall, beta-d-glucan (BDG), may be a useful diagnostic marker for fungal infections. In a recent study comparing colonized patients to those with IC, BDG was superior to blood cultures in identifying deep-seated candidiasis. The combination of blood cultures and BDG had a sensitivity of $79 \%$, suggesting that BDG may be a useful adjunct to blood cultures, to aid in the identification of some patients who are currently 
undiagnosed [6]. Our study aims to determine the incidence and type of Candida colonization and infection in SICU, as well as to relate the observed BDG concentrations to patients' fungal status.

\section{Methods}

Screening

The study was carried out in a tertiary care hospital in Singapore over a three-month period (November 2010 and January to February 2011). Patients above 18 years of age, who were admitted to Singapore General Hospital (SGH) surgical intensive care unit (SICU) for at least $48 \mathrm{~h}$, were prospectively identified from Electronic Health Records (Eclipsys Sunrise, Allscripts Corp., Chicago, IL). The following patients were excluded from the study: aged younger than 18; neutropenic (defined as absolute neutrophil count $<0.5 \times 10^{9} / \mathrm{L}$ ); pregnant/lactating; or were colonized/had documented or possible fungal infections as diagnosed by an infectious diseases specialist (including fungal infections other than Candida spp.) prior to SICU admission .

This study was approved by the SingHealth Centralized Institutional Review Board (IRB no. 2010/494/D) and waiver of consent was granted.

\section{Surveillance cultures}

Surveillance cultures for Candida spp. were obtained from eligible patients, with the first samples taken on the third day post ICU admission, and weekly thereafter until discharge from the ICU or death. This corresponded to the days which BDG levels were determined. The following sites were cultured for fungal microorganisms: i) rectum (i.e. rectal swabs or feces), ii) urine (if any), iii) respiratory tract (e.g. bronchoalveolar lavage specimens), iv) wound (if any), and v) blood. Results were considered positive in the presence of Candida growth in the culture medium. The different Candida isolates were further speciated at the microbiology laboratory.

All included patients would be eventually classified into three groups with respect to their fungal status: 1) neither colonized nor infected, 2) Candida species colonization without IC, and 3) Candida species colonization with proven IC.

Colonization was considered unifocal when Candida species were isolated from one site and considered multifocal when Candida species were simultaneously isolated from various noncontiguous foci, even if two different Candida species were isolated.

\section{Diagnosis of Candida infections}

Proven IC will require one of the following criteria: 1) Histologically confirmed candidiasis from a specimen obtained by needle aspiration or biopsy from a normally sterile site (other than mucous membranes); or 2)
Isolation of Candida species in a sample obtained by a sterile procedure (including a freshly placed $[<24 \mathrm{~h}$ ago $]$ drain) from a normally sterile site in a patient with consistent clinical manifestations; or 3) documentation of one or more blood culture(s) that yielded a Candida species in a patient with consistent clinical manifestations [7].

Candidal peritonitis is defined as the isolation of Candida species in a peritoneal sample obtained by laparotomy, including perforation of an abdominal organ, dehiscence of an intestinal suture with peritonitis, severe acute pancreatitis, or presence of peritoneal catheter for dialysis. Catheter-related candidemia will be considered in patients who have an intravascular device and one or more positive cultures of blood samples obtained from the peripheral vein, clinical manifestations of infection (e.g., fever, chills, and/or hypotension), and no apparent source for bloodstream infection (with the exception of the catheter), as well as a positive catheter culture. Candiduria is defined as the presence of at least $10^{4}$ colony-forming units/mL of the same Candida species.

The decision to treat a patient with antifungal drugs during the course of this study was entirely dependent on the attending physician's clinical judgment. For all study patients on antifungal agents, details of therapy as well as clinical and microbiological information were collected.

\section{BDG assay}

Serum samples were collected from all eligible patients on (i) the third day of ICU admission and (ii) once a week there after until ICU discharge, death or occurrence of IC.

Blood samples $(15 \mathrm{~mL})$ were collected in three tubes without anticoagulant, centrifuged at $1800 \mathrm{rpm}$ for $10 \mathrm{~min}$, separated into aliquots, and stored at $-80{ }^{\circ} \mathrm{C}$ until analysis. Detection of BDG was performed using Fungitell assays (Associates of Cape Cod Incorporated, MA, US) in duplicates by the serology and immunology laboratory in our institution according to the manufacturer's instructions. Negative controls (blood from healthy volunteers) were included. Results were regarded as valid if both duplicates were in the same category (both positive and negative) and duplicates did not differ by more than $20 \%$. Detection of positive BDG is defined as BDG level of $\geq 80 \mathrm{pg} / \mu \mathrm{L}$.

\section{Data collection}

The following variables were recorded for each patient: age, gender, date of ICU admission, dates of ICU and hospital discharge, reason for ICU admission, underlying diseases, concomitant infections, number and sites of Candida colonization and risk factors (treatment with corticosteroids with a daily dose $\geq 20 \mathrm{mg}$ prednisolone for at least 2 weeks, use of broad spectrum antibiotics or 
antimicrobials within 10 days before the study, use of mechanical ventilation before the day of inclusion in the study, and urinary catheter in place on the day of inclusion). Type of surgery (abdominal vs non-abdominal, elective vs urgent) and the number of major procedures performed before and during ICU stay were registered. Acute Physiology and Chronic Health Evaluation (APA$\mathrm{CHE}$ ) II and Charlson comorbidity index were also recorded at ICU admission.

\section{Candida score and Candida colonization index}

Candida score (CS) and colonization index (CI) were calculated on the days whenever BDG levels were determined. According to Pittet's definitions, the CI was defined as the ratio of the number of distinct non-blood body sites colonized by Candida species to the total number of body sites cultured [8]. Patients with $\mathrm{CI} \geq 0.5$ were considered heavily colonized. The CS was calculated as follows (variables coded as absent $=0$, present $=1$ ): total parenteral nutrition $\times 1$, plus surgery $\times 1$, plus multifocal Candida colonization $\times 1$, plus severe sepsis $\times 2$. A CS $\geq 3$ accurately selected patients at high risk for IC.

\section{Anti-fungal susceptibility testing}

Minimum inhibitory concentrations (MICs) of amphotericin B, fluconazole, voriconazole, anidulafungin, caspofungin and micafungin were tested for each non-duplicate isolate using the Etest method performed in accordance to manufacturer's recommendations (bioMérieux, Marcy l'Etoile, France). Briefly, Etests were carried out on plates containing RPMI agar supplemented with $2 \%$ glucose and buffered to pH 7.0 with morpholinepropanesulfonic acid (Sigma-Aldrich, St Louis, MO, US). The inoculum (0.5 McFarland-adjusted cell suspension in $0.85 \% \mathrm{NaCl}$ ) was swabbed in three directions on the entire RPMI-agar plate, and the E-test strip was applied after excess moisture had been absorbed into the agar. The plates were incubated at $35{ }^{\circ} \mathrm{C}$, and MICs were read after $24 \mathrm{~h}$.

MICs were interpreted in accordance to Clinical and Laboratory Standards Institute (CLSI) species-specific clinical breakpoints, where available [8]. Epidemiological cut-off values were used when breakpoints are not proposed by CLSI.

\section{Statistical analysis}

Categorical variables were expressed as frequencies and percentages, and continuous variables as medians and range. Kruskal Wallis and Chi-square (or Fisher's exact) tests were used to analyze continuous and categorical variables, respectively. Sensitivity and specificity were calculated for BDG, Candida score and CI. Receiver operator curves (ROC) was used to compare sensitivity and specificity between assays.

\section{Results}

\section{Study population}

Out of 187 patients admitted to SICU during the study period, 155 patients were excluded because of lack of fulfillment of the inclusion criteria (Fig. 1). Only 28 patients met the criteria for recruitment. Characteristics of SICU patients are presented in Table 1. They had a median age of 62 (32-85) years old. Upon SICU admission, the median APACHE II score was 20 (5-42) and Charlson Co-morbidity index was $5(0-16)$. The median length of ICU stay was 6.5 (3-71) days. Nineteen patients underwent emergency operations; three patients underwent elective operations while six patients did not have any operations. Fourteen patients had abdominal surgery while the remaining 8 patients had non-abdominal related surgeries. There were two patients staying beyond one week in SICU stay and had a second BDG assay done. The APACHE II score, number of risk factors, CI (a second round of surveillance swabs were done) and CS were re-calculated for these two patients, when second sets of BDG assays were performed. Hence, there were 30 cases (with BDG) classified for analysis in this study.

There were three $(10.0 \%)$ patients who were neither colonized nor infected. Twenty-three $(76.7 \%)$ patients were colonized without infection. Of these, five patients were colonized at one site and 18 at two or more sites. Three patients with multi-focal colonization and one patient with unifocal colonization developed IC.

Out of the three patients with multi-focal colonization, two had disseminated Candida infections involving multiple sites. Patient one was admitted into SICU for severe sepsis post-brevis flap surgery. A CT abdominal pelvis scan was done and new pancreatic collections were observed. Drainage was performed and the fluid was cultured, isolating C. tropicalis. In addition, C. tropicalis and $C$. albicans were also isolated from her blood. Anti-

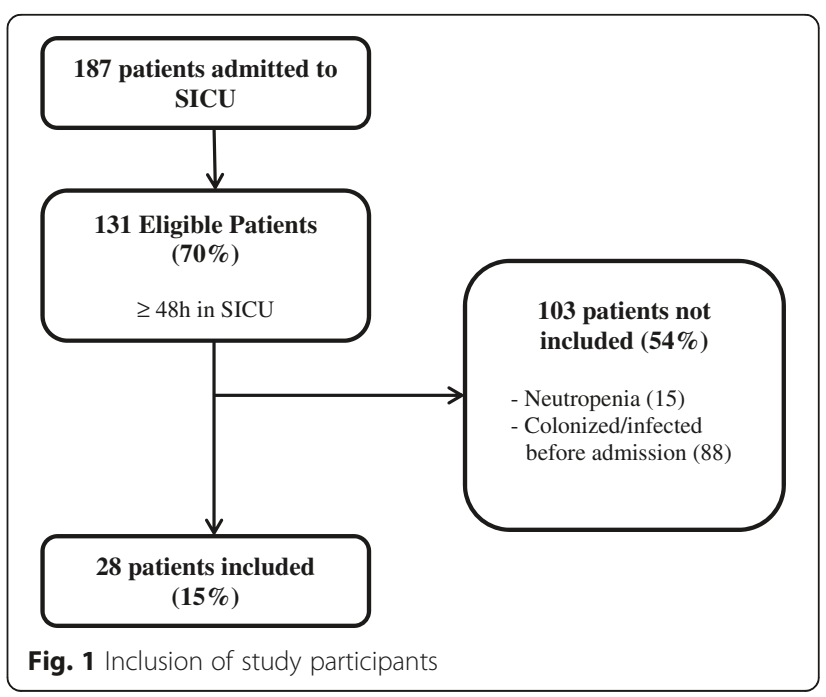


Table 1 Characteristics of SICU patients

\begin{tabular}{llllll}
\hline $\begin{array}{l}\text { Colonization } \\
\text { Status }\end{array}$ & $\begin{array}{l}\text { No. of } \\
\text { cases }\end{array}$ & Age & $\begin{array}{l}\text { Apache II } \\
\text { Score }\end{array}$ & $\begin{array}{l}\text { Charlson } \\
\text { Comorbidity } \\
\text { Score }\end{array}$ & $\begin{array}{l}\text { No of risk } \\
\text { factors }\end{array}$ \\
\hline $\begin{array}{l}\text { Colonized \& } \\
\text { Infected }\end{array}$ & -4 & 69 & 22 & 4 & 4 \\
$\begin{array}{l}\text { Colonized \& Non-infected } \\
\text { Unifocal }\end{array}$ & 5 & 63 & 16 & 4 & 2 \\
$\quad$ Multifocal & 18 & 62 & 26 & 6 & 2 \\
Non-colonized & 3 & 57 & 16 & 4 & 2 \\
\hline
\end{tabular}

fungal treatment (fluconazole) was started five days later when the blood culture result was available and subsequently treatment was changed to caspofungin. Finally, the patient underwent an exploratory laparotomy with cholecystectomy, pancreatic necrotomy and further drainage of intra-abdominal collections. She demised within a week post-operatively from acute pancreatitis. Patient two, who had underlying adenocarcinoma of the colon, underwent an exploratory laparotomy for a perforated duodenal ulcer. Intraoperative findings included ulcer perforation and gross peritonitis with C. albicans isolated from the peritoneal fluid and blood. Immediately post-surgery, the patient deteriorated rapidly, and demised four days after. Anti-fungal therapy was not instituted as the culture and susceptibilities result were not reported in time. The third patient with multi-focal colonization was admitted for septic shock due to a perforated antral ulcer which required an emergency partial gastrectomy. In week one of ICU stay, his peritoneal fluid then grew Candida non-albicans, for which he was treated with fluconazole upon the availability of culture result. The patient improved and was discharged from SICU. For the patient with unifocal colonization, he was admitted to SICU for septic shock secondary to acute cholangitis. Within $24 \mathrm{~h}$ post-ERCP, C. albicans was isolated from the bile, obtained via a bile drain placed intra-operatively, and his IC episode was subsequently treated with fluconazole when culture result was available. The patient improved and was discharged from SICU.

Five cases received empiric anti-fungal therapy after a new onset of fever while receiving broad-spectrum antibiotics and none of them had culture-proven candidiasis. Two patients underwent intra-abdominal surgeries (exploratory laparotomies) for ischemic bowel resection and intestinal obstruction. One patient had acute necrotizing pancreatitis with cholangiocarcinoma, and another patient had an above knee amputation done, which was complicated with worsening acidosis post-surgery. One patient had intra-abdominal sepsis secondary to abscess which was drained, but received no intra-abdominal surgery. The median (range) time to administration of anti-fungals from onset of sepsis was five days (4-18). Four patients received echinocandins while the remaining one received fluconazole for empiric therapy. These five patients had BDG median readings of 108.33 (46.16276.23) $\mathrm{pg} / \mu \mathrm{L}$, with median $\mathrm{CI}$ of 0.67 (0.33-0.75), and median CS of $3(3-4)$. Two of the five patients died (the patient with acute necrotizing pancreatitis and the patient who had an above knee amputation).

\section{BDG levels}

The BDG levels attained by the patients are presented in Table 2. Ten BDG levels were negative with median reading of $45.01(14.95-68.20) \mathrm{pg} / \mu \mathrm{L}$ while 20 BDG levels were positive at median reading of 189.45 (90.65->500) $\mathrm{pg} / \mu \mathrm{L}$, including the 4 patients with IC. The median BDG value in group 1, 2 and 3 were 112.14 (90.65-201.47) pg/ $\mu \mathrm{L}, 104.58(14.95->500) \mathrm{pg} / \mu \mathrm{L}$, and $338.72(124.62->500) \mathrm{pg} / \mathrm{uL}$ respectively. There was no significant statistical difference between the median BDG values in the three groups $(p=0.07)$. Only two patients had serial BDG assay done due to prolonged stay (more than one week) in SICU. On day three of SICU admission, one patient had elevated BDG reading of $>500 \mathrm{pg} / \mu \mathrm{L}$ with CI of 1and CS of 4 , while the other patient had low BDG reading of $28.84 \mathrm{pg} / \mu \mathrm{L}$ with $\mathrm{CI}$ of 0.5 and CS of 5 . On day seven of SICU admission, the patient with elevated BDG continued to have elevated BDG reading of $>500 \mathrm{pg} / \mu \mathrm{L}$ and $\mathrm{CI}$ of 1 . Blood culture was now positive, confirming the diagnosis of IC. The CS had increased to five. The patient with low BDG reading continued to have low BDG reading of $43.86 \mathrm{pg} /$ $\mu \mathrm{L}$ with $\mathrm{CI}$ of 0.5 and CS of 3 .

\section{$\mathrm{Cl}$ and $\mathrm{CS}$}

Eight of the calculated CI was $<0.5$ while 22 of the calculated CI was $\geq 0.5$. Nineteen patients $(82.6 \%)$ had CI $\geq 0.5$ and 4 patients $(17.4 \%)$ with $\mathrm{CI}$ of $<0.5$ in group 2 . Three patients $(75.0 \%)$ in group 3 had CI $\geq 0.5$. There was significant statistical difference between the three groups $(p=0.02)$.

Table 2 BDG levels, $\mathrm{Cl}$ and CS in different groups

\begin{tabular}{|c|c|c|c|c|c|c|c|}
\hline \multirow{3}{*}{$\begin{array}{l}\text { Colonization } \\
\text { Status }\end{array}$} & \multirow{3}{*}{$\begin{array}{l}\text { No. } \\
\text { of } \\
\text { cases } \\
\text { (N) }\end{array}$} & \multirow{3}{*}{$\begin{array}{l}\text { Median BDG } \\
(\mathrm{pg} / \mu \mathrm{l})\end{array}$} & \multicolumn{5}{|c|}{ No of cases $(\mathrm{N})$} \\
\hline & & & \multicolumn{3}{|c|}{$\begin{array}{l}\text { Median BDG } \\
\text { Range (pg/ul) }\end{array}$} & \multirow[t]{2}{*}{$\begin{array}{l}\mathrm{Cl} \geq \\
0.5\end{array}$} & \multirow[t]{2}{*}{$\begin{array}{l}\mathrm{CS} \geq \\
3\end{array}$} \\
\hline & & & $<60$ & $\begin{array}{l}60- \\
80\end{array}$ & $>80$ & & \\
\hline $\begin{array}{l}\text { Colonized \& } \\
\text { Infected }\end{array}$ & 4 & 338.72 & 0 & 0 & 4 & 3 & 4 \\
\hline \multicolumn{8}{|c|}{ Colonized \& Non-infected } \\
\hline Unifocal & 5 & 108.33 & 1 & 0 & 4 & 1 & 5 \\
\hline Multifocal & 18 & 85.58 & 6 & 3 & 9 & 18 & 15 \\
\hline Non-colonized & 3 & 112.14 & & & 3 & 0 & 2 \\
\hline
\end{tabular}


Four CS were $<3$ while 26 CS were $\geq 3$. Two patients in group 1 had $C S \geq 3$ while 1 patients had $C S<3$. Twenty patients had $C S \geq 3$ while 3 patients had $C S<3$ in group 2 . All patients $(100 \%)$ in group 3 had CS of $\geq 3$. There was no significant statistical difference between the three groups $(p=0.6)$.

\section{Comparison of BDG, $\mathrm{Cl}$ and CS for diagnosis of IC}

Table 3 summarizes the diagnostic accuracy of the BDG values versus $\mathrm{CI}$ and $\mathrm{CS}$ in the diagnosis of IC. All three tests had good sensitivity (75-100\%) but poor specificity. BDG fared the best in terms of specificity at $38.46 \%$ while CS had the poorest specificity at $15.38 \%$. In view of the low specificity of $\mathrm{CS}$, we recalculated the sensitivity and specificity at a cut-off of 4 . This increased the specificity to $53.85 \%$ but sensitivity fell to $50 \%$. The ROCs of BDG, CI and CS are presented in Fig. 2. BDG performed the best with an AUC of 0.89 .

\section{Candida surveillance cultures and anti-fungal susceptibility}

The results of the surveillance cultures for Candida spp. are presented in Table 4. Each positive swab could yield more than one Candida isolate. Of 136 swabs cultured from different anatomic sites of 28 SICU patients, 52 (38.2\%) were positive for Candida spp. These isolates included C. albicans $(\mathrm{n}=32), C$. glabrata $(\mathrm{n}=27), C$. dubliniensis $(\mathrm{n}=11), C$. tropicalis $(\mathrm{n}=17)$ and $C$. parasilopsis $(\mathrm{n}=3)$ (Table 2$)$. Twelve patients $(40 \%)$ had more than one type of Candida species from the various sites and specimens tested. Overall, the frequency of Candida isolation is highest in the rectum $(80 \%)$, followed by sputum (74\%), urine (35\%), wound (33\%) and blood (6\%). The antifungal susceptibilities are presented in Table 5. Azole resistance was detected in C. albicans (7 \%). C. glabrata and C. tropicalis were, respectively, 100 and $7 \%$ SDD to fluconazole.

\section{Discussion}

ICU patients are at higher risk for IC than patients who are in the general ward [9]. Among the known risk factors for IC, Candida colonization is probably the most important one as it indicates that patients have an endogenous source of candida [10]. It was noteworthy that all recruited patients in this study were immunocompetent and, $73 \%$ had $\mathrm{CI} \geq 0.5$ upon first surveillance; with
Candida albicans as the predominant species isolated in the surveillance cultures. Given that this study is conducted in the SICU, it is not surprising that most of the patients had candida scores $\geq 3$. Interestingly, despite the high CI and CS scores indicating the higher risk for IC in these patients, only 4 cases of IC were detected in this study. This suggests that relying on either CI or CS alone may over predict for IC in these patients and lead to unnecessary use of antifungal agents.

In this study, 5 out of 14 patients with intra-abdominal sepsis received empiric antifungal treatment and all were colonized (CI 0.33 to 0.75 ) with BDG levels ranging from 46 to $276 \mathrm{pg} / \mu \mathrm{l}$. Empiric antifungals were generally initiated within 7 days of ICU stay, after patients had received a range of wide-spectrum antibiotics and yet did not improved clinically. Unfortunately, only 2 out of the 14 patients with intra-abdominal sepsis survived, underscoring the high case-fatality rates and thus, the need for earlier detection of occult invasive candidiasis and institution of antifungal treatment. One well-known strategy is the preemptive approach relying on fungal biomarkers e.g. betaD-glucan.

We evaluated the use of BDG, CS and CI to aid earlier detection of patients with suspected IC. Presently, BDG is widely used as a diagnostic marker for invasive fungal infections and is included as indirect microbiological criteria for the diagnosis of probable IFI in the revised EORTC definitions [7]. This was based on the results from studies evaluating the use of BDG to select patients with candida colonization and through serial readings, to guide preemptive antifungals patients in these high risk patients $[11,12]$.

Using the FDA approved cut-off of $\geq 80 \mathrm{pg} / \mu \mathrm{l}$, Mohr reported a BDG sensitivity and specificity of 91 and $57 \%$ respectively [13]. A more recent study by Tissot reported sensitivity and specificity of 83 and $52 \%$ in patients with culture-negative intraabdominal sepsis [14]. These results are relatively comparable to those observed in this study where the sensitivity was $100 \%$ and specificity was $40 \%$. Our lower specificity could be contributed by the high number of false positives in our patient cohort. The median BDG of the 3 non-colonized and non-infected patients was $112.14 \mathrm{pg} / \mu \mathrm{l}$ (Range $91-125 \mathrm{pg} / \mu \mathrm{L}$ ), above the manufacturer's cutoff value of $80 \mathrm{pg} / \mu \mathrm{l}$. This anomaly may be explained by the fact all non-colonized patients underwent intra-abdominal surgery which is a surrogate

Table 3 Accuracy of BDG, Cl and CS in diagnosing IC

\begin{tabular}{lllll}
\hline & Sensitivity (\%) & Specificity (\%) & PPV (\%) & NPV (\%) \\
\hline BDG $>80$ & $100(40.23-100)$ & $38.46(20.25-59.42)$ & $20.00(5.86-43.67)$ & $100(68.97-100.00)$ \\
$C l \geq 0.5$ & $75(20.34-95.88)$ & $26.92(11.62-47.79)$ & $13.64(3.06-34.94)$ & $87.50(47.38-97.93)$ \\
$C S \geq 3$ & $100(40.23-100)$ & $15.38(4.45-34.84)$ & $15.38(4.45-34.89)$ & $100(40.23-100)$ \\
$C S \geq 4$ & $50.00(8.30-91.70)$ & $53.85(33.39-73.39)$ & $14.29(2.20-42.84)$ & $87.50(61.62-98.08)$ \\
\hline
\end{tabular}




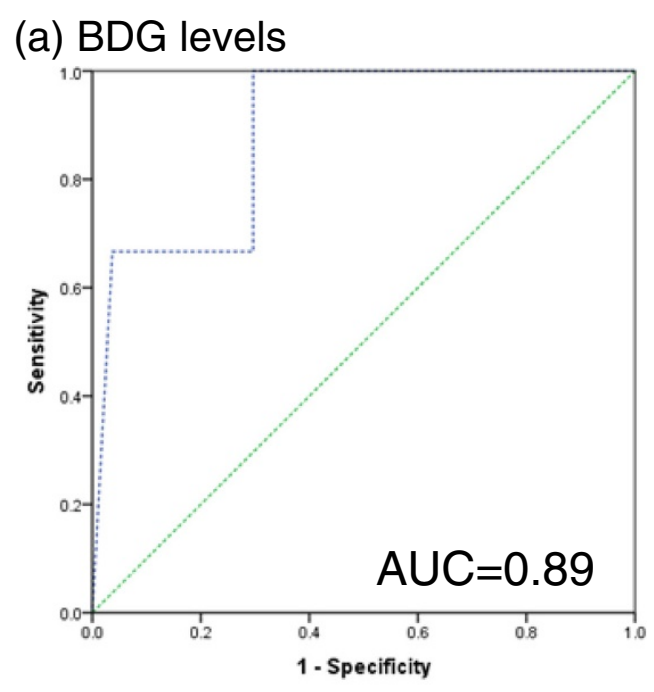

(b) $\mathrm{Cl}$

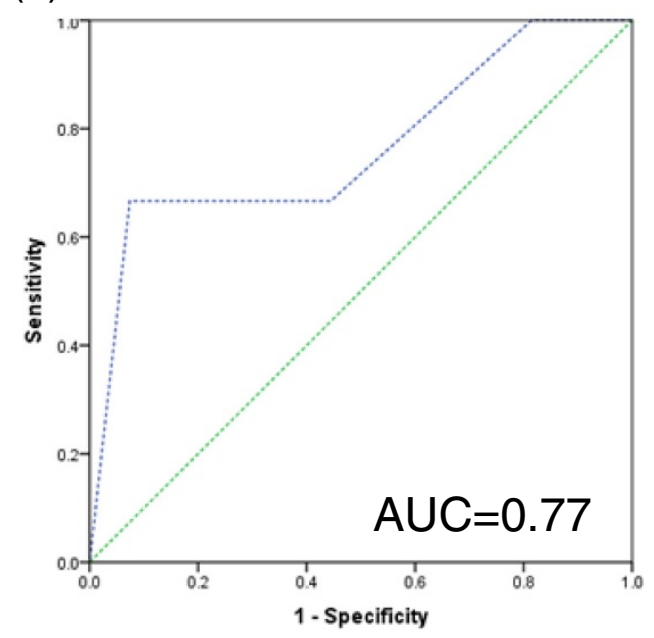

(c) CS

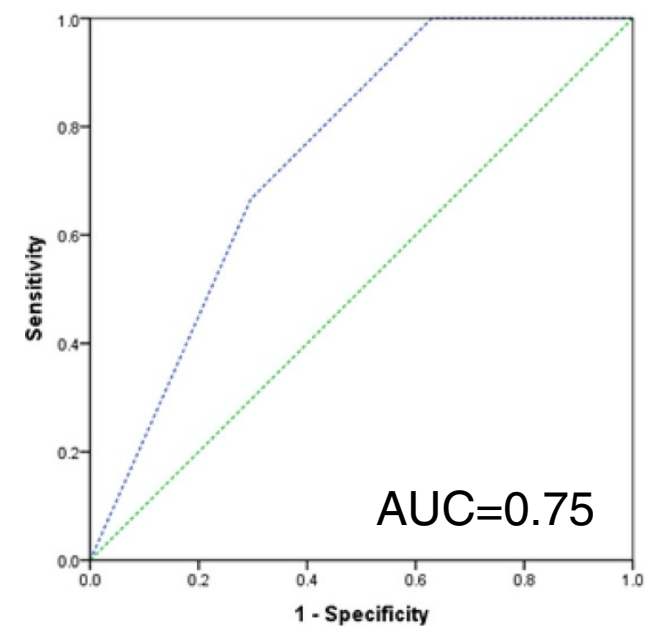

Fig. 2 ROC for differentiating IC using (a) BDG levels; (b) Cl; (c) CS
Table 4 Species spectrum of Candida species isolated from different anatomic sites of SICU patients

\begin{tabular}{llllll}
\hline Site & C. & C. & C. & C. & C. \\
& albicans & dubliniensis & glabrata & tropicalis & parasilopsis \\
\hline Blood & 1 & 0 & 2 & 1 & 0 \\
Urine & 3 & 0 & 2 & 0 & 0 \\
Rectal & 13 & 6 & 14 & 7 & 2 \\
Sputum & 14 & 5 & 8 & 9 & 1 \\
Wound & 1 & 0 & 1 & 0 & 0 \\
Total & 32 & 11 & 27 & 17 & 4 \\
\hline
\end{tabular}

for the use of surgical gauzes; a known cause of falsely elevated BDG [15]. Abdominal surgery involving transection of the gastrointestinal tract also increases the potential for dissemination of BDG in the bloodstream. Additionally, 2 out of 3 of these patients had concurrent bacterial infections, another potential cause of reactivity with the BDG assay [15]. These observations concur with that reported by Tissot et al. where 87 and $62 \%$ of non-colonized patients in their study cohort with false-positive BDG had concurrent bacteremia and abdominal surgery respectively [14].

In the SICU, the high proportion of patients who received abdominal surgery may possibly render the use of BDG readings to "rule in" IC inefficient. On the contrary, using the BDG to "rule out" IC may be more applicable given its high negative predictive value observed in this and previous studies. A prospective pilot study on pre-emptive anidulafungin using BDG by Hanson reported similar PPV and NPV of 20.7 and $100 \%$ respectively [11]. As PPV and NPV are influenced by disease prevalence, we believe that this was due to the similar low IC rates observed in our studies. Serial BDG readings $>80 \mathrm{pg} / \mu \mathrm{l}$ had been suggested to increase the specificity of this test without compromising on the NPV [11]. Furthermore, trending of serial BDG levels and subsequent observation of a decreasing gradient was associated with successful echinocandin therapy in one study. Baseline and post-treatment may potentially be useful as prognostic markers of treatment outcome in patients with IC receiving primarily echinocandin therapy [16]; as well as serve as a guide to safely discontinue empiric antifungal treatment in culture-negative ICs. As most of our study patients stayed in the ICU for less than a week, we were unfortunately unable to evaluate these hypotheses.

Although not statistically significant, the median BDG values for infected IC patients were higher than those for colonized and non-colonized patients. A similar pattern was reported by Tissot where the median BDG in the patients with proven IC $(223 \mathrm{pg} / \mathrm{ml})$ was significantly higher than the other groups, including those with suspected and treated IC [14]. As such, a markedly 
Table 5 Antifungal susceptibilities

\begin{tabular}{|c|c|c|c|c|c|c|}
\hline Candida species & Anti-fungal & MIC 50 & MIC Range & $\% \mathrm{~S}$ & $\% S D D / l$ & $\% \mathrm{R}$ \\
\hline C. albicans & Fluconazole & 0.380 & $0.064-\geq 512$ & 87 & 7 & 7 \\
\hline \multicolumn{7}{|l|}{$(n=15)$} \\
\hline & Voriconazole & 0.008 & $0.008-\geq 64$ & 87 & 7 & 7 \\
\hline & Anidulafungin & 0.003 & $\leq 0.002-0.012$ & 100 & 0 & 0 \\
\hline & Caspofungin & 0.016 & $\leq 0.002-0.064$ & 100 & 0 & 0 \\
\hline & Micafungin & 0.012 & $0.002-0.032$ & 100 & 0 & 0 \\
\hline & Amphotericin B & 0.064 & $\leq 0.002-0.250$ & 100 & - & 0 \\
\hline \multirow{7}{*}{$\begin{array}{l}\text { C. glabrata } \\
(n=14)\end{array}$} & Fluconazole & 8 & $0.125-24$ & - & 100 & 0 \\
\hline & & & & & & \\
\hline & Voriconazole & 0.125 & $0.004-0.75$ & - & - & - \\
\hline & Anidulafungin & 0.032 & $\leq 0.002-0.125$ & 100 & 0 & 0 \\
\hline & Caspofungin & 0.032 & $\leq 0.002-0.125$ & 100 & 0 & 0 \\
\hline & Micafungin & 0.016 & $0.004-0.032$ & 100 & 0 & 0 \\
\hline & Amphotericin B & 0.125 & $\leq 0.002-0.250$ & 100 & - & 0 \\
\hline \multirow{7}{*}{$\begin{array}{l}\text { C. tropicalis } \\
(n=9)\end{array}$} & Fluconazole & 1.5 & $0.38-4$ & 89 & 11 & 0 \\
\hline & & & & & & \\
\hline & Voriconazole & 0.064 & $0.016-0.38$ & 67 & 33 & 0 \\
\hline & Anidulafungin & 0.006 & $\leq 0.002-0.094$ & 100 & 0 & 0 \\
\hline & Caspofungin & 0.032 & $\leq 0.002-0.190$ & 100 & 0 & 0 \\
\hline & Micafungin & 0.023 & $0.016-0.032$ & 100 & 0 & 0 \\
\hline & Amphotericin B & 0.190 & $\leq 0.002-0.250$ & 100 & 0 & 0 \\
\hline \multirow{7}{*}{$\begin{array}{l}\text { C. parapsilosis } \\
(n=2)\end{array}$} & Fluconazole & - & $0.25-0.5$ & 100 & 0 & 0 \\
\hline & & & & & & \\
\hline & Voriconazole & - & $0.012-0.023$ & 100 & 0 & 0 \\
\hline & Anidulafungin & - & $0.38-0.75$ & 100 & 0 & 0 \\
\hline & Caspofungin & - & $0.38-0.75$ & 100 & 0 & 0 \\
\hline & Micafungin & - & $0.25-0.5$ & 100 & 0 & 0 \\
\hline & Amphotericin B & - & $0.064-0.5$ & 100 & - & 0 \\
\hline \multirow{7}{*}{$\begin{array}{l}\text { C. dubliniensis } \\
(\mathrm{n}=8)\end{array}$} & Fluconazole & 0.5 & $0.125-\geq 512$ & - & - & - \\
\hline & & & & & & \\
\hline & Voriconazole & 0.023 & $0.004-\geq 64$ & - & - & - \\
\hline & Anidulafungin & 0.006 & $\leq 0.002-0.032$ & - & - & - \\
\hline & Caspofungin & 0.016 & $\leq 0.002-0.094$ & - & - & - \\
\hline & Micafungin & 0.047 & $0.012-0.094$ & - & - & - \\
\hline & Amphotericin B & 0.006 & $\leq 0.002-0.25$ & - & - & - \\
\hline
\end{tabular}

elevated single BDG is strongly indicative of proven IC and was even shown to be associated with poor clinical outcome [17]. In our study, 1 of the 2 patients with BDG $>500 \mathrm{pg} / \mu \mathrm{l}$ died of IC.

The BDG, CI and CS appear largely similar in their sensitivities, specificities, PPVs and NPVs. When the ROCs of the three modalities were analyzed, BDG provided the highest AUC of 0.89. It may be that the use of serial BDG levels may further increase the specificity and AUC correspondingly for detection of IC.
The main limitation of this study was the small number of subjects with proven IC as mentioned earlier. Despite liberal inclusion criteria, we had a difficult time recruiting patients not already receiving systemic antifungal therapy by day 3 of ICU admission.

\section{Conclusion}

Colonization with Candida species in surgical patients is common, and can result in positive BDG test. BDG, CS and CI demonstrated high sensitivities but poor 
specificities in this study population. Given their high NPV, their utilities may lie in excluding IC instead. In particular, the kinetics of BDG in serial readings may instead be used in antifungal stewardship programs to guide discontinuation of empiric (culture-negative) antifungal treatments Nevertheless, BDG performed the best (AUC of 0.89) when compared with CS and CI in our study. Our findings need further validation.

\section{Competing interests}

The authors declare that they have no competing interests.

\section{Authors' contributions}

JT carried out analysis of fungal susceptibility data and assist in the analysis of the rest of the data. IAT participated in recruitment of patients. ALT was involved in all microbiological testing of all clinical samples. CCN was involved in beta-d-glucan testing of samples. WL participated in the design of the study, performed the statistical analysis, and vetted/ amended the manuscript. ALK conceived of the study, participated in its design and vetted/amended the manuscript. MPC provided expert opinion in all treatment during treatment of all patients. YXL helped in coordination, performed the statistical analysis and helped to draft the manuscript. All authors read and approved the final manuscript.

\section{Acknowledgements}

This research was supported by Singapore General Hospital Research Grant 2010. This publication was supported in part by the SGH Centre Grant from the National Medical Research Council, Singapore (ref: NMRC/CG/016/2013)

\section{Author details}

'Department of Pharmacy, Singapore General Hospital, Block 8 Level 2, Outram Road, Singapore 169608, Singapore. ${ }^{2}$ Division of Nursing, Department of SICU, Singapore General Hospital, Outram Road, Singapore 169608, Singapore. ${ }^{3}$ Department of Pathology, Singapore General Hospital, Outram Road, Singapore 169608, Singapore. ${ }^{4}$ Department of Infectious Diseases, Singapore General Hospital, Outram Road, Singapore 169608, Singapore. ${ }^{5}$ Emerging Infectious Diseases, Duke-National University of Singapore Graduate Medical School, 8 College Rd, Singapore 169857, Singapore. ${ }^{6}$ Department of Pharmacy, Faculty of Science, National University of Singapore, Block S4A, Level 3, 18 Science Drive 4, Singapore 117543, Singapore.

Received: 27 August 2014 Accepted: 23 June 2015

Published online: 03 July 2015

\section{References}

1. Falagas ME, Apostolou KE, Pappas VD. Attributable mortality of candidemia: a systematic review of matched cohort and case-control studies. Eur J Clin Microbiol Infect Dis. 2006;25(7):419-25.

2. Wey SB, Mori M, Pfaller MA, Woolson RF, Wenzel RP. Hospital-acquired candidemia. The attributable mortality and excess length of stay. Arch Intern Med. 1988;148(12):2642-5.

3. Pfaller M, Neofytos D, Diekema D, Azie N, Meier-Kriesche HU, Quan SP, et al. Epidemiology and outcomes of candidemia in 3648 patients: data from the Prospective Antifungal Therapy (PATH Alliance') registry, 2004-2008. Diagn Microbiol Infect Dis. 2012;74(4):323-31.

4. Beck-Sague C, Jarvis WR. Secular trends in the epidemiology of nosocomial fungal infections in the United States, 1980-1990. National Nosocomial Infections Surveillance System. J Infect Dis. 1993;167(5):1247-51.

5. Berenguer J, Buck M, Witebsky F, Stock F, Pizzo PA, Walsh TJ. Lysis-centrifugation blood cultures in the detection of tissue-proven invasive candidiasis. Disseminated versus single-organ infection. Diagn Microbiol Infect Dis. 1993;17(2):103-9.

6. Nguyen MH, Wissel MC, Shields RK, Salomoni MA, Hao B, Press EG, et al. Performance of Candida real-time polymerase chain reaction, beta-D-glucan assay, and blood cultures in the diagnosis of invasive candidiasis. Clin Infect Dis. 2012;54(9):1240-8.

7. De Pauw B, Walsh TJ, Donnelly JP, Stevens DA, Edwards JE, Calandra T, et al. Revised definitions of invasive fungal disease from the European
Organization for Research and Treatment of Cancer/Invasive Fungal Infections Cooperative Group and the National Institute of Allergy and Infectious Diseases Mycoses Study Group (EORTC/MSG) Consensus Group. Clin Infect Dis. 2008;46(12):1813-21.

8. CLSI. Clinical and Laboratory Standard Institute. Reference Method for Broth Dilution Antifungal Susceptibility Testing of Yeasts; 4th Informational Supplement. PA, USA: 2012. https://clsi.org/.

9. Wisplinghoff $H$, Bischoff $T$, Tallent SM, Seifert $H$, Wenzel RP, Edmond MB. Nosocomial bloodstream infections in US hospitals: analysis of 24,179 cases from a prospective nationwide surveillance study. Clin Infect Dis. 2004;39(3):309-17.

10. Miranda LN, van der Heijden IM, Costa SF, Sousa AP, Sienra RA, Gobara S, et al. Candida colonisation as a source for candidaemia. J Hosp Infect. 2009;72(1):9-16.

11. Hanson KE, Pfeiffer CD, Lease ED, Balch AH, Zaas AK, Perfect JR, et al. beta-D-glucan surveillance with preemptive anidulafungin for invasive candidiasis in intensive care unit patients: a randomized pilot study. PLoS One. 2012;7(8):e42282.

12. Takesue $Y$, Kakehashi M, Ohge H, Imamura Y, Murakami Y, Sasaki M, et al. Combined assessment of beta-D-glucan and degree of candida colonization before starting empiric therapy for candidiasis in surgical patients. World J Surg. 2004;28(6):625-30.

13. Mohr JF, Sims C, Paetznick V, Rodriguez J, Finkelman MA, Rex JH, et al. Prospective survey of $(1 \rightarrow 3)$-beta-D-glucan and its relationship to invasive candidiasis in the surgical intensive care unit setting. J Clin Microbiol. 2011;49(1):58-61.

14. Tissot F, Lamoth F, Hauser PM, Orasch C, Fluckiger U, Siegemund M, et al. beta-glucan antigenemia anticipates diagnosis of blood culture-negative intraabdominal candidiasis. Am J Respir Crit Care Med. 2013;188(9):1100-9.

15. Albert O, Toubas D, Strady C, Cousson J, Delmas C, Vernet V, et al. Reactivity of $(1 \rightarrow 3)$-beta-d-glucan assay in bacterial bloodstream infections. Eur J Clin Microbiol Infect Dis. 2011;30(11):1453-60.

16. Jaijakul S, Vazquez JA, Swanson RN, Ostrosky-Zeichner L. (1,3)-beta-D-glucan as a prognostic marker of treatment response in invasive candidiasis. Clin Infect Dis. 2012;55(4):521-6.

17. Rhein J, Bahr NC, Morawski BM, Schutz C, Zhang Y, Finkelman M, et al. Detection of High Cerebrospinal Fluid Levels of $(1 \rightarrow 3)-\beta$-d-Glucan in Cryptococcal Meningitis. Open Forum Infect Dis. 2014;1(3):ofu105.

\section{Submit your next manuscript to BioMed Central and take full advantage of:}

- Convenient online submission

- Thorough peer review

- No space constraints or color figure charges

- Immediate publication on acceptance

- Inclusion in PubMed, CAS, Scopus and Google Scholar

- Research which is freely available for redistribution 